proportion of patients in categories A, B, C and D, respectively, when evaluated by CAT was 10, 49, 1 and $40 \%$, and when evaluated by $\mathrm{mMRC}$ was $39,20,13$ and $28 \%$. By CAT evaluation in categories $A, B, C$, and $D$, patients were using a long-acting $\beta_{2}$-agonist (LABA) alone (8, 6, 0 and 1\%), long-acting muscarinic antagonist (LAMA) alone $(37,25,8$ and $5 \%$ ), inhaled corticosteroid plus LABA (ICS/ LABA) alone (22, 18, 8 and $8 \%$ ), and ICS/LABA plus LAMA only $(11,20,46,43 \%)$.

Conclusion CAT assessment increased the number of patients in the more symptomatic categories ( $\mathrm{B}$ and $\mathrm{D})$, compared with mMRC. Contrary to the GOLD 2011 recommendations, by CAT assessment, a high proportion of low-risk patients ( $\mathrm{A}$ and $\mathrm{B}$ ) were using ICS/LABA.

\section{P186 PERSPECTIVES OF PATIENT AND PROFESSIONAL PARTICIPANTS ON TELEHEALTHCARE AND THE IMPACT ON SELF-MANAGEMENT: OUALITATIVE STUDY NESTED IN THE TELESCOT COPD TRIAL}

doi:10.1136/thoraxjnl-2012-202678.247

${ }^{1} \mathrm{H}$ Pinnock, 'P Fairbrother, ${ }^{2} \mathrm{~J}$ Hanley, ${ }^{1} \mathrm{~L}$ McCloughlan, ${ }^{1} \mathrm{~A}$ Todd, 'B McKinstry. ${ }^{1}$ The University of Edinburgh, Edinburgh, UK; ${ }^{2}$ Edinburgh Napier University, Edinburgh, UK

Background The TELESCOT randomised control trial, is investigating the impact of a telemonitoring service for COPD with the primary aim of reducing hospitalisation.

Aim The nested qualitative study explored the views of patients and professionals on models of telemetric service delivery and the impact on self-management.

Method Semi-structured interviews with patient and professional participants at different time points in the TELESCOT trial were transcribed, coded and analysed thematically. Interpretation was supported by multidisciplinary discussion.

Results 38 patients ( $47 \%$ male, mean age 67.5 years) and 32 healthcare professionals provided 70 interviews. Both patients and professionals considered that home telemonitoring had the potential to reduce the risk of hospital admission.

Patients generally appreciated being 'watched over' by the telemonitoring, which gave them confidence to manage their own condition. They used tele-data to improving their understanding of COPD, determine their current state of health and influence decisions about their daily activities. Numerical data (e.g. oxygen saturations) were particularly valued. Changes in readings validated their decisions to adjust treatment or seek timely professional advice, and eased access to clinical care.

Professionals emphasised the potential role of telemetry in encouraging prompt compliance with medically defined behaviours and attitudes, though there was concern that 'fixation' on monitoring physiological parameters (especially oxygen saturation levels), promoted a medical model of the disease and might increase dependence on services in some patients.

The GPs and community nursing or physiotherapy teams who provided home telemonitoring support services emphasised the importance of 'knowing the patient' and 'knowing what's normal for the individual' in using their clinical skills to interpret incoming telemonitoring data.

Conclusion Enthusiasm for telemonitoring as a means of facilitating self-management and thereby reducing admissions is tempered by concerns about increased medicalisation and dependence on support services. Tele-monitoring provides data which can be used to support self-management decisions and act as a channel for seeking professional support. The patient-practitioner relationship, personalisation and continuity of care were prioritised as important elements in delivering clinical support for telemonitoring services by patients and professionals.

Funding Chief Scientist Office, Scottish Government.

\section{P187 TREATMENT OF STABLE COPD IN ALPHA 1 ANTITRYPSIN DEFICIENCY (AATD) PATIENTS USING THE 2011 GOLD TREATMENT ALGORITHM}

doi:10.1136/thoraxjnl-2012-202678.248

A Pillai, RA Stockley. University Hospitals, Birmingham, UK

Background Previous versions of the GOLD strategy algorithm recommended treatment based on FEV1 which is now recognised as a poor descriptor of disease impact. The revised 2011 GOLD document has introduced individualised assessment of symptoms and future risk for initial management of stable COPD. Questionnaires such as COPD Assessment Test (CAT) and modified Medical Research Council (mMRC) are suggested to assess symptoms and risk depends on spirometric impairment or exacerbation frequency. Aim To apply the current GOLD treatment algorithm to AATD patients and assess their current treatment against that recommended.

Methods 309 consecutive patients on the AATD registry (PiZZ) were grouped into the four categories (A, B, C, and D) as suggested by GOLD, on the basis of their CAT 10, 13 or mMRC scores and GOLD spirometric stage or Exacerbation frequency. We then documented the treatment combinations being used by the patients in the different groups.

Results Treatment for patients in the four groups differed widely from GOLD recommendations. Few patients in Group A(CAT10GOLD/MRCGOLD/CAT13GOLD:6\%/2\%/2\% and CAT10EXAC/MRCEXAC/CAT13EXAC:6\%/2\%/3\%) were on the GOLD recommended SABA/SAMA therapy. $40 \%$ were on none and $30 \%$ were on triple therapy. The majority of patients in group $B$ and Group C (60\% and 58\% respectively) were on triple therapy with only $10 \%$ and $14 \%$ respectively being on the recommended regimen. Nearly $70 \%$ in group D were on recommended triple therapy with ICS, LABA and LAMA.

The proportion of patients in the groups $A, B$ and $C$ assessed by mMRC was significantly different using CAT $10(p<0.02)$ but not using CAT 13 ( $p=$ not significant). The proportion of patients in group D was similar for all 3 symptom scores ( $p=$ not significant). Conclusions CAT 13 was most comparable to mMRC for patient distribution. Most of the AATD patients with low risk (low symptoms and high symptoms) were over treated with triple therapy. The majority in the high risk/high symptoms group were appropriately on triple therapy. It remains to be determined how this affects long term outcome.

\section{P188 CAT SCORE THRESHOLD FOR SYMPTOM/RISK ASSESSMENT IN ALPHA-1-ANTITRYPSIN DEFICIENCY (AATD) USING THE 2011 GOLD ALGORITHM}

doi:10.1136/thoraxjnl-2012-202678.249

A Pillai, RA Stockley. University Hospitals, Birmingham, UK

Background The revised COPD GOLD guidelines (2011) recommend that validated questionnaires like the CAT (COPD Assessment Test) or the modified MRC (Medical Research Council) breathlessness scale should be used to assess symptoms in COPD against "risk" as assessed by GOLD spirometric staging or exacerbation frequency. We have demonstrated that when either the MRC or CAT scores are used to determine symptoms, there is a significant difference in the proportion of patients being categorised into the risk categories which will affect risk assessment and may influence therapeutic choice.

Aim To determine the CAT threshold for risk assessment at which similar proportions of patients are categorised into the 4 risk categories.

Methods 309 consecutive patients on the AATD registry (PiZZ) were grouped into the four categories (A, B, C, and D) as suggested 